EPJ Web of Conferences 52, 02002 (2013)

DOI: 10.1051/epjconf/20135202002

(C) Owned by the authors, published by EDP Sciences, 2013

\title{
An empirical model to describe rapidity density and transverse momentum distributions
}

\author{
Akinori Ohsawa $^{1, a}$, Edison H. Shibuya ${ }^{2, b}$, and Masanobu Tamada ${ }^{3, c}$ \\ ${ }^{1}$ Institute for Cosmic Ray Research, University of Tokyo, Kashiwa, 277-8582 Japan \\ ${ }^{2}$ Instituto de Fisica Gleb Wataghin, Universidade Estadual de Campinas, Campinas, 13083-970, Brasil \\ ${ }^{3}$ Faculty of Science and Engineering, Kinki University, Higashi-Osaka, 577-8502 Japan
}

\begin{abstract}
The distribution of rapidity density and transverse momentum is formulated empirically and analytically. It describes the data quite well over the wide energy range of $\sqrt{s}=22.4-7000 \mathrm{GeV}$.
\end{abstract}

\section{Introduction}

We formulate the transverse momentum and rapidity density distributions of produced particles in multiple particle production (MPP) empirically and analytically. Compared with the current models which use a theoretical idea and the simulation technique, present model has following advantages.

(1) It describes all existing data concerned reasonably.

(2) Consequently it is the most suitable one to be used in analyzing high energy cosmic-ray events.

(3) We can see the least necessary ingredients to describe the MPP.

\section{Transverse momentum and rapidity density distributions}

\subsection{Assumptions}

We enumerate the assumptions to formulate the rapidity density distribution, $d^{2} N_{c h} / d y^{*} d p_{T}$, of produced particles in this section, the details of which are found in Ref.[1].

(1) All produced particles are pions. (The assumption affects slightly the rapidity density distribution.)

(2) Produced particles are emitted isotropically from several centers.(Fig. 1) The emitting centers are distributed on the rapidity axis in the center of mass system (CMS) with the distribution $g\left(y^{\prime}\right) d y^{\prime}$ of

$g\left(y^{\prime}\right)= \begin{cases}c & \left(0 \leq y^{\prime}<b y_{0}\right) \\ c-\frac{c(1-d)}{y_{0}(1-b)}\left(y^{\prime}-b y_{0}\right) & \left(b y_{0} \leq y^{\prime}<y_{0}\right) \\ 0 & \left(y^{\prime} \geq y_{0}\right)\end{cases}$

where the values of the parameters $b=d=0.25$ are determined by trial and error by fitting the (pseudo-)rapidity

\footnotetext{
ae-mail: ohsawa@icrr.u-tokyo.ac.jp

be-mail: shibuya@if.unicamp.br

ce-mail: tamada@ele.kindai.ac.jp
}

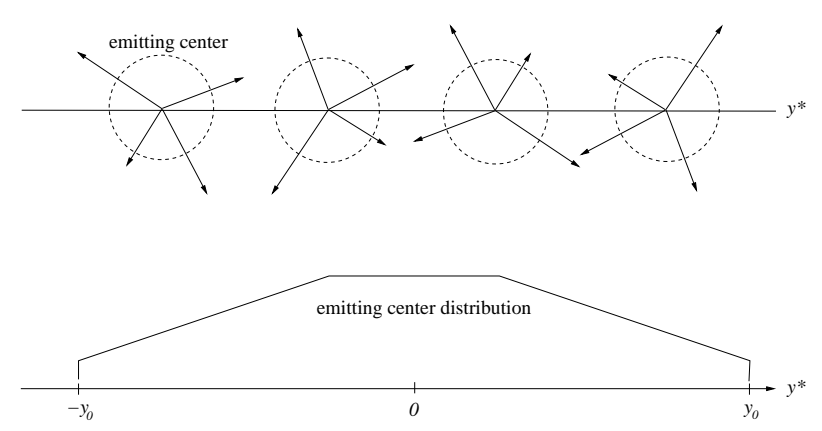

Figure 1. Distribution of the emitting centers on the rapidity axis in CMS. The parameter $y_{0}$ is defined as $y_{0}=\ln (\sqrt{s} / M)-\ln a_{2}$ where $M$ is nucleon mass and $a_{2}$ an adjustable parameter. (See 2.1.) The produced particles are emitted isotropically from several emitting centers. The energy distribution of produced particles is that of Tsallis type, eq.(2), on respective emitting centers.

distributions to the data at various energies. The parameter $c=2 / y_{0}[2 b+(1-b)(1-d)]$ is determined by the normalization of the distribution. The parameter $y_{0}$, defined as $y_{0}=\ln (\sqrt{s} / M)-\ln a_{2}(M:$ nucleon mass), is determined through fitting the (pseudo-)rapidity density distributions to the data at various energies by adjusting the value of the parameter $a_{2}$.

(3) The energy distribution of produced particles $f(p) d p$ is that of Tsallis type on the respective emitting centers. That is,

$$
f(p)=C \frac{p^{2}}{T_{0}^{3}}\left[1+\frac{q-1}{T_{0}} p\right]^{-q /(q-1)} \quad(q>1)
$$

where the values of the parameters $T_{0}$ and $q$ are determined so as to reproduce the transverse momentum (abbreviated as $p_{T}$ hereafter) distributions at various energies.

Then the rapidity density distribution is

$$
\frac{d^{2} N_{c h}}{d y^{*} d p_{T}}=a_{1} y_{0} \int_{-y_{0}}^{y_{0}} \frac{p_{T} E}{2 p^{2}} f(p) g\left(y^{\prime}\right) d y^{\prime}
$$


where $E=\mu \cosh \left(y^{*}-y^{\prime}\right), p=\sqrt{E^{2}-m^{2}}, \mu=\sqrt{p_{T}^{2}+m^{2}}$ ( $m$ : pion mass). (The quantity with an asterisk is the one in CMS.) The distribution can be converted to the pseudo-rapidity density distribution and $x$ distribution $\left(x^{*} \equiv 2 p_{\|}^{*} / \sqrt{s}\right)$ easily.

\section{$2.2 p_{T}$ distributions}

The $p_{T}$ distribution at the zenith angle $\theta^{*}=90^{\circ}$, expressed in terms of the invariant cross section, is

$$
\begin{gathered}
\left.E \frac{d^{3} \sigma}{d^{3} p}\right|_{\theta^{*}=90^{\circ}}=\frac{\sigma_{\text {inel }}}{2 \pi p_{T}}\left(\frac{d^{2} N_{c h}}{d y^{*} d p_{T}}\right)_{y^{*}=0} \\
=\frac{\sigma_{\text {inel }}}{4 \pi} a_{1} y_{0} \int_{-y_{0}}^{y_{0}} \frac{\mu \cosh \left(y^{\prime}\right)}{p^{2}} f(p) g\left(y^{\prime}\right) d y^{\prime}
\end{gathered}
$$

where $p=\sqrt{\left[\mu \cosh \left(y^{\prime}\right)\right]^{2}-m^{2}}$. The distribution includes three parameters $T_{0}, q$ and $a_{1}$ since the integration is almost independent of the parameter $a_{2}$ at high energies, i.e. $\ln (\sqrt{s} / M) \gg 3$. We calculate the $p_{T}$ distributions for several pairs of values of the parameters $\left(T_{0}, q\right)$ to look for the best fitting one to the data. The value of the parameter $a_{1}$ can be obtained through the empirical relation of the quantity $\rho_{0}$, the pseudo-rapidity density at $\eta^{*}=0$,

$$
\begin{array}{r}
\rho_{0} \equiv \int_{0}^{\infty}\left(\frac{d^{2} N_{c h}}{d \eta^{*} d p_{T}}\right)_{\eta^{*}=0} d p_{T} \\
=2.716-0.307 \ln (s)+0.0267 \ln ^{2}(s)
\end{array}
$$

obtained by CMS Collaboration[2], since it includes the parameters $T_{0}, q$ and $a_{1}$. The distributions describe the $p_{T}$ distribution well over the whole region of the data an an incident energy. (See Fig. 2.)

The values of the parameters $T_{0}$ and $q$ in the energy distribution are obtained by fitting the $p_{T}$ distributions to the data at the energies of $\sqrt{s}=$ $63,200,500,900,1800,2360,7000 \mathrm{GeV}$. The empirical energy dependences of them are

$$
\begin{array}{r}
q=0.0295 \log _{10}\left(\frac{\sqrt{s}}{1.0 \mathrm{GeV}}\right)+1.020, \\
T_{0}= \begin{cases}0.122 & \left(\sqrt{s} \leq \epsilon_{0}\right) \\
0.0163 \log _{10}\left(\frac{\sqrt{s}}{1.0 \mathrm{GeV}}\right) & \left(\sqrt{s}>\epsilon_{0}\right)\end{cases}
\end{array}
$$

with $\epsilon_{0}=3.82 \times 10^{2} \mathrm{GeV}$.

\subsection{Pseudo-rapidity density distributions}

The pseudo-rapidity distribution

$$
\frac{d^{2} N_{c h}}{d \eta^{*} d p_{T}}=\frac{p_{T}\left(e^{\eta^{*}}+e^{-\eta^{*}}\right)}{\sqrt{p_{T}^{2}\left(e^{\eta^{*}}+e^{-\eta^{*}}\right)^{2}+4 m^{2}}} \frac{d^{2} N_{c h}}{d y^{*} d p_{T}}
$$

includes four parameters of $T_{0}, q, a_{1}$ and $a_{2}$. The former two have been determined already and the latter two are determined by fitting the distributions to the data. The distributions describe the data well over the whole region of the data at any incident energy. (See Fig. 3.)

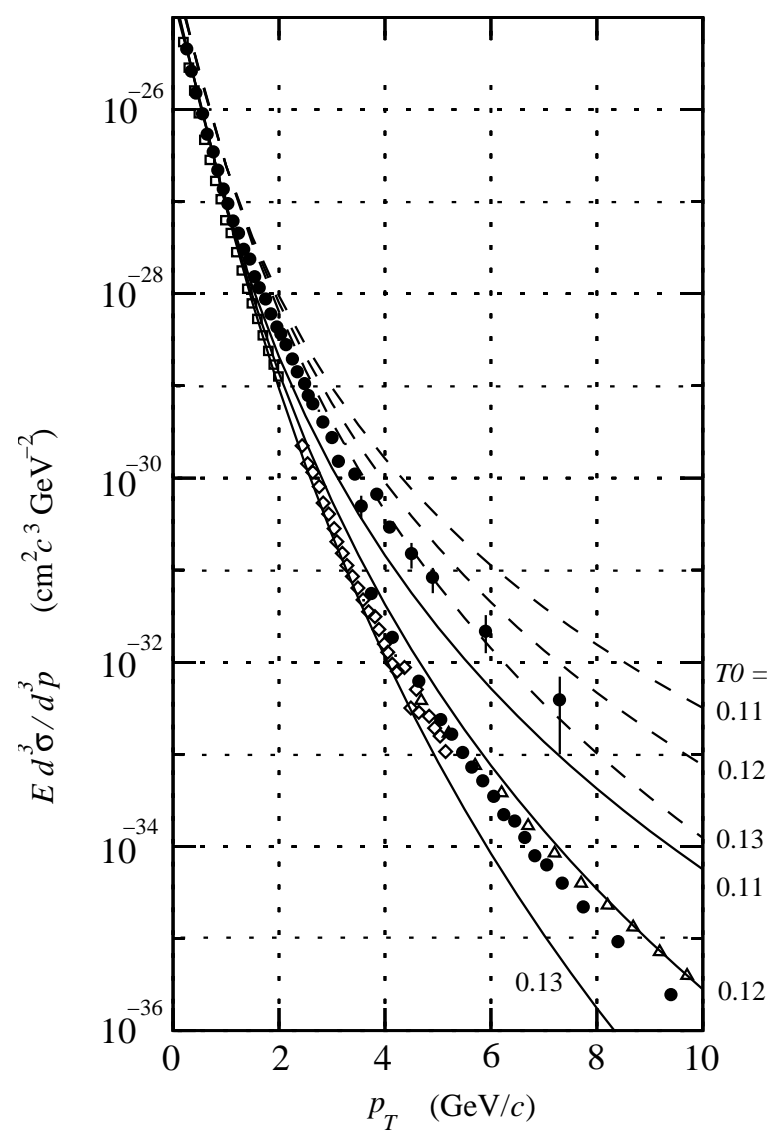

Figure 2. The $p_{T}$ distributions at $\sqrt{s}=63$ (lower)[3] and 500 (upper) $\mathrm{GeV}$ [4]. Experimental data are for a half of the charged hadrons (or $\pi^{0}$ s) and hence the curves are a half of eq.(4). The solid lines (at $63 \mathrm{GeV}$ ) and chain lines (at $500 \mathrm{GeV}$ ) are for the values of the parameters in the table below. The values of $q$ are determined so as to reproduce the average $p_{T}$ 's at respective energies for the values of $T_{0}$.

\begin{tabular}{crc}
\hline $\begin{array}{c}T_{0} \\
(\mathrm{GeV} / c)\end{array}$ & $\sqrt{s}=63 \mathrm{GeV}$ & $\stackrel{q}{s}=500 \mathrm{GeV}$ \\
\hline 0.11 & 1.098 & 1.128 \\
0.12 & 1.075 & 1.109 \\
0.13 & 1.055 & 1.090 \\
\hline
\end{tabular}

The best fitting curves are for the values $(0.122,1.071)$ and $(0.125,1.100)$ of the parameters $\left(T_{0}, q\right)\left(T_{0}\right.$ in $\left.\mathrm{GeV} / c\right)$ at $\sqrt{s}=63$ $\mathrm{GeV}$ and $500 \mathrm{GeV}$, respectively.

The values of the parameters $a_{1}$ and $a_{2}$ are obtained by fitting the pseudo-rapidity density distributions to the data at the energies of $\sqrt{s}=$ $22.4,200,540,630,900,1800,2360,7000 \mathrm{GeV}$. The empirical energy dependences of them are

$a_{1}=1.85\left(\frac{\sqrt{s}}{100 \mathrm{GeV}}\right)^{0.225}, a_{2}=0.940\left(\frac{\sqrt{s}}{10 \mathrm{GeV}}\right)^{\alpha}$

The two exponents of the parameter $a_{2}$ are for the maximum and minimum dependences since the values of $a_{2}$ are 
distributed widely due to the limited data in the forward region of the rapidity.

\section{Discussions}

Present model describes the rapidity density and $p_{T}$ distributions quite well over the whole region of the data in the wide energy range of $\sqrt{s}=22.4-7000 \mathrm{GeV}$. Consequently it reproduces the energy dependence of the average $p_{T}$, that of the charged multiplicity and that of the pseudo-rapidity density at $\eta^{*}=0$. It describes the $p_{T}$ averages at the rapidity $y^{*}=5.01-6.59$ in the forward region, obtained by UA7 Collaboration[6] at $\sqrt{s}=630 \mathrm{GeV}$, too.

The inelasticity, expected by the model, either increases or decreases with the energy from the assumed

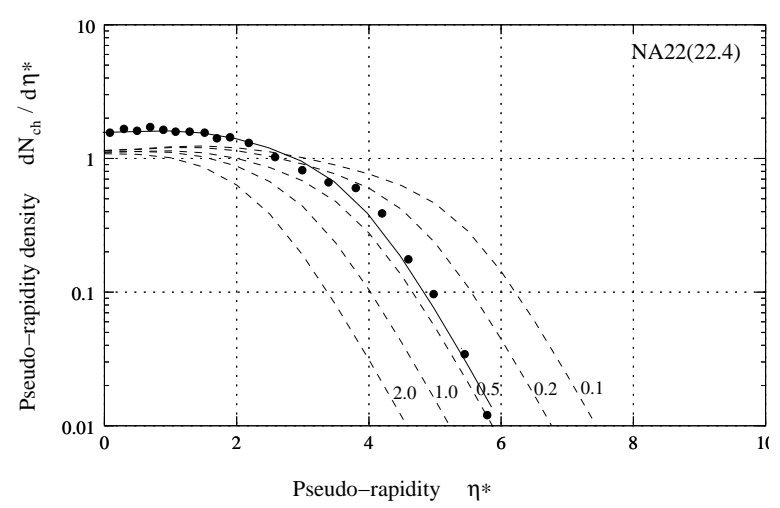

Figure 3. Pseudo-rapidity density distribution at $\sqrt{s}=22.4$ $\mathrm{GeV}$. The chain lines are for the value of the parameters $a_{1}=1.0$ and $a_{2}=0.1,0.2,0.5,1.0,2.0$ (attached to the curves). The solid curve of $a_{1}=1.38$ and $a_{2}=0.5$ is the best fitting one to the data by EHS-NA22 Collaboration.[5] value of $K=0.5$ at $\sqrt{s}=10 \mathrm{GeV}$ for the minimum and maximum dependences of the parameter $a_{2}$ (See 2.3.), i.e. $K=0.95$ and 0.18 at $\sqrt{s}=10^{5} \mathrm{GeV}$, respectively.

\section{References}

[1] A. Ohsawa et al., Intern. Journ. Mod. Phys. A 27 No.9 1250043 (2012).

[2] V. Khachatryan et al. (CMS Collab.), Phys. Rev. Lett. 105, 022002 (2010).

[3] G. Arnison et al., Phys. Lett. B118, 173 (1982).

[4] C. Albajar et al., Nucl. Phys. B335, 261 (1990).

[5] M. Adamus et al., Z. Phys. C39, 311 (1988).

[6] E. Pare et al., Phys. Lett. B242, 531 (1990). 